\title{
Molecular Dynamics Simulation of Saposin C-Membrane Binding
}

\author{
Anping Liu ${ }^{1}$ and Xiaoyang $\mathrm{Qi}^{*}, 2$ \\ ${ }^{1}$ Department of Chemistry, University of Cincinnati, Cincinnati, OH 45221-0172, USA \\ ${ }^{2}$ Division and Program in Human Genetics, Cincinnati Children's Hospital Research Foundation, University of Cincin- \\ nati College of Medicine, 3333 Burnet Avenue, Cincinnati, Ohio 45229-3039, USA
}

\begin{abstract}
To understand the mechanism of Saposin C-membrane binding at low $\mathrm{pH}$, several molecular dynamics (MD) simulations of saposin C with 1,2-dioleoyl-sn-glycero-3-phosphatidylserine (DOPS 18; 1) or modified DOPS were performed in the default charge states at neutral $\mathrm{pH}$ or, based on the $\mathrm{pKa}$ calculations, with some protonated acidic residues to approximate low $\mathrm{pH}$ conditions. It was found that the electrostatic attraction between the protein- $\mathrm{COO}^{-}$and lipid- $\mathrm{NH}_{3}{ }^{+}$ groups played an important role in the binding process in addition to the electrostatic attraction between the lipid- $\mathrm{COO}^{-}$ and protein- $\mathrm{NH}_{3}{ }^{+}$groups. The two pairs of attractive interactions acted basically on the opposite sites of saposin C. Neutralization of the amino groups of the lipids enabled saposin C-binding with the membrane at neutral $\mathrm{pH}$. The simulation results provide insight into the nature and mechanism of the Saposin C-membrane binding process at low $\mathrm{pH}$.
\end{abstract}

Keywords: $\mathrm{MD}$, protein-membrane interaction, saposin $\mathrm{C}$, phosphatidylserine, low $\mathrm{pH}$ binding.

\section{INTRODUCTION}

Saposin $\mathrm{C}$ is a member of the saposin family. Saposins are important activators of selected enzymes that cleave sphingolipids in lysosomes. Their structures and functions have been intensively studied [1-11]. Although they have differential interactions with phospholipid bilayers, saposins have the general property of binding lipid membranes. Interestingly, a conformational change is observed in 1,2dioleoyl-sn-glycero-3-phosphatidylserine (DOPS 18; 1) membrane-bound saposin $\mathrm{C}$ using a fluorescence spectroscopic method [12]. This protein structural alteration induced by DOPS is important for saposin C's biological activity. Similarly, a structural change when saposin $\mathrm{C}$ binds sodium dodecyl sulfate (SDS) was determined by NMR [13].
[14]. All charged amino acid side chains in saposin $\mathrm{C}$ are solvent exposed. The electrostatic surface of saposin $\mathrm{C}$ in Fig. (1b) shows that the protein has a predominantly negative potential (shown in red). The few isolated positive potentials (shown in blue) correspond to the Lys residues located on the surface of the $\mathrm{H}-1$ and $\mathrm{H}-2$ helices. The binding of saposin $\mathrm{C}$ to phospholipid vesicles was found to be a $\mathrm{pH}-$ controlled reversible process and many solvent-exposed glutamate residues have elevated $\mathrm{pKa}$ values close to the binding $\mathrm{pKa}$ of 5.3. The conclusion was that the partial neutralization of the negatively charged electrostatic surface of saposin $\mathrm{C}$ triggered membrane binding. Lipid degradation in lysosomes was anticipated to be switched on and off by saposin C's reversible binding to membrane [14].

To better understand the mechanism of saposin C's bind-

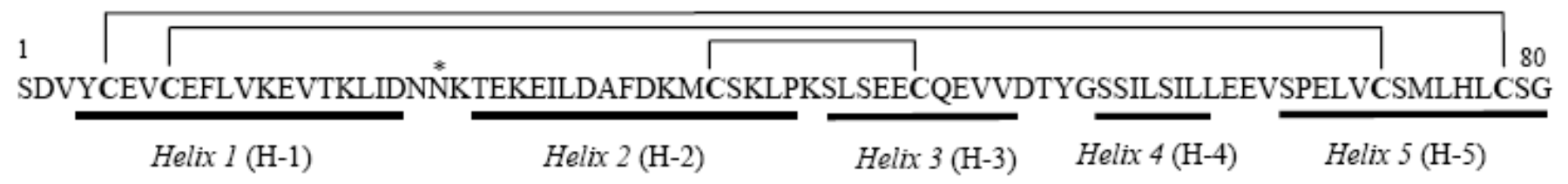

Fig. (1a). Amino acid sequence of saposin C. The sequence of saposin C shows the N-glycosylation site (asterisk) and the 6 cysteines (C, boldface) to form three disulfide bonds. The $\alpha$-helical regions are also indicated.

Saposin C, illustrated in Fig. (1a), is a small protein $(\sim 80$ AA) with an N-glycosylation sequence (asterisk) and 6 cysteines $(\mathrm{C}$, boldface). The three disulfide bridges and locations of the $\alpha$-helices (H-1 to H-5) are also shown in Fig. (1a). The three-dimensional structure of saposin $\mathrm{C}$ (Protein databank code; 1M12) has been determined by NMR study

*Address correspondence to this author at the Division and Program in Human Genetics, Cincinnati Children's Hospital Research Foundation, University of Cincinnati College of Medicine, Cincinnati, Ohio 45229-3039, USA; Tel: (513) 636-5964; Fax: (513) 636-3486; E-mail: qix0@chmcc.org ing with phospholipid membranes at the molecular level, we performed molecular dynamics simulations of saposin C, first with the default residue charge states and then with several neutralized acidic residues to mimic the low $\mathrm{pH}$ condition in the negatively charged DOPS membrane. Since the charge states of the ionizable residues have a great impact on the property and conformation of the protein, a series of $\mathrm{pKa}$ calculations for saposin $\mathrm{C}$ with different protein dielectric constants at different $\mathrm{pH}$ were also carried out. The neutralized residues to be used in the simulation were determined from the $\mathrm{pKa}$ calculations. The change of the $\mathrm{pH}$ value was found to regulate the attractive interactions between the 


\section{Front view}

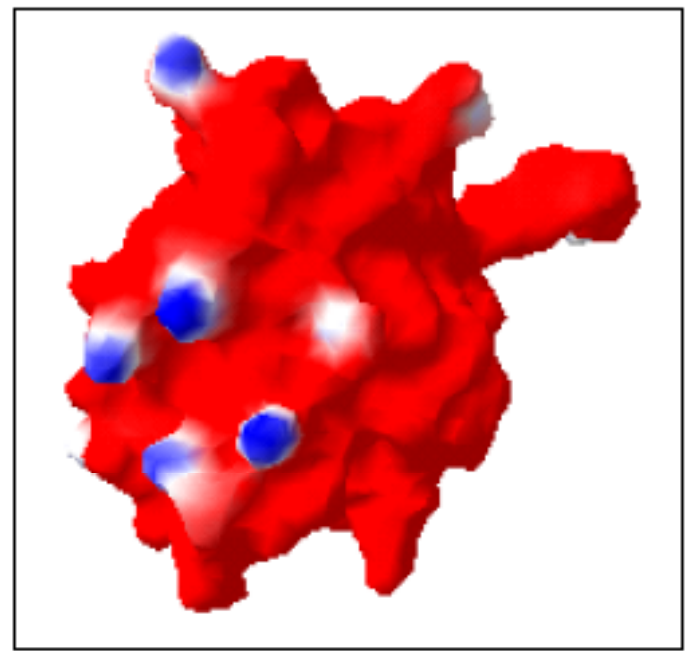

Back view

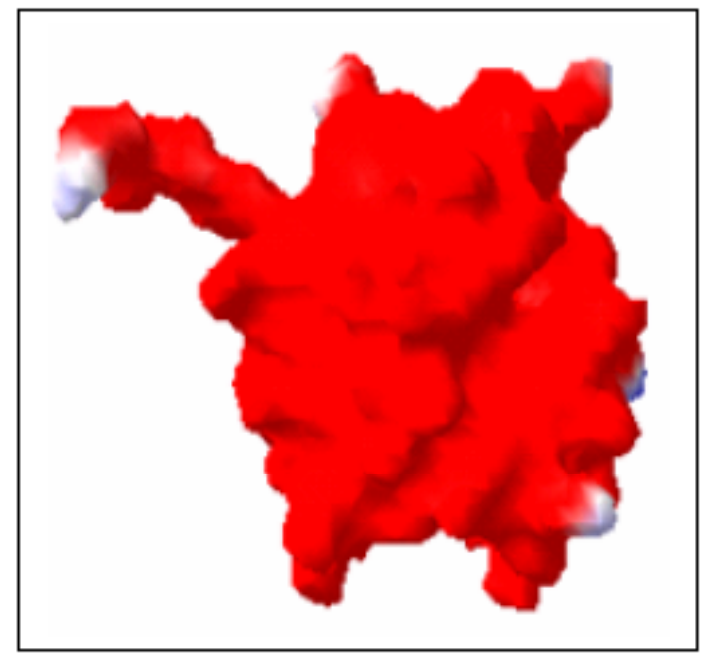

Fig. (1b). Front and back views of the electrostatic surface of saposin C. Red color represents a negative potential and blue color represents a positive potential. Graphics were prepared using the Deep View/Swiss-Pdb Viewer.

negatively charged Glu residues of the protein with the positively charged amino termini of the lipids. The repulsive interactions between the negatively charged residues of the protein with the negatively charged carboxylate termini of the lipids played a less important role in saposin Cmembrane binding. The relative position of saposin $\mathrm{C}$ to the membrane is mainly determined by two pairs of attractive interactions, i.e. between the negatively charged residues of the protein with the positively charged amino termini of the lipids and the positively charged Lys residues of the protein with the negatively charged carboxylate termini of the lipids. In general, as shown in Fig. (1b), these two pairs of different attractive interactions act on the opposite surfaces of saposin C. The negative-negative repulsion prevents the back side surface (mainly H3-H5) of saposin $\mathrm{C}$ to bind membrane. The protein-membrane binding is realized through the interactions of the protein Lys residues located on the front side surface (mainly H1-H2) with the carboxylate groups of the membrane, but is controlled by the other pair of attractive interactions. Experiments also found that saposin $\mathrm{C}$ bound to the detergent SDS at neutral $\mathrm{pH}$ and was $\mathrm{pH}$ independent [13]. However, SDS does not have positively charged head groups, so there are no attractive interactions between the Glu and Asp residues of saposin C with SDS.

In the present study, we conclude that the $\mathrm{pH}$ mainly controls the electrostatic interaction between the protein- $\mathrm{COO}^{-}$ and lipid- $\mathrm{NH}_{3}{ }^{+}$groups, which determines whether saposin C's Lys side chains can closely approach the membrane surface and bind with the membrane. Simulation results agree with recent experimental results by others [13], support our conclusion regarding the mechanism of the saposin C/DOPS binding, and provide insight into the nature and mechanism of the saposin C-membrane binding process at low $\mathrm{pH}$.

\section{METHODS}

The structure of saposin $\mathrm{C}$ was obtained from the Protein Data Bank with accession code 1M12. The University of Houston Brownian Dynamics (UHBD) program [15], ver- sion 5.1, was used for $\mathrm{pKa}$ calculations of saposin C. In the UHBD program, a continuum dielectric model (Single Site Model) with a detailed atomic description of the protein was used [16]. The dielectric constant of the solvent was assigned the value of 80 . There is a large amount of literature on what dielectric constant value in the protein should be assigned to yield the best results for $\mathrm{pKa}$, anywhere from 2 to 80 [1723].

The $\mathrm{pKa}$ calculations in this paper were carried out at the protein dielectric constants of 2, 4, 6, 10, 20, and 80 in the range of $\mathrm{pH} \sim 4.5-7.0$. The dielectric boundary was determined from the solvent accessible surface with a probe radius of $1.4 \AA$ and the dielectric constant was smoothed at the adjacent grid points. The ionic strength of the solvent was assumed to be $150 \mathrm{mM}$ and to follow a Boltzmann distribution at $298 \mathrm{~K}$. The molecule was surrounded by a $2 \AA$ ion exclusion layer. For the calculation of the electric potential of each titratable site, four cubic grids with spacing of 2.5, $1.2,0.75$, and $0.25 \AA$ and $45^{3}, 15^{3}, 15^{3}$, and $20^{3}$, respectively, were used.

The saposin C structure in a DOPS monolayer was simulated with an all-atom CHARMM27 force field of CHARMM (Chemistry at Harvard Macromolecular Mechanics) and NAMD (Not Another Molecular Dynamics) [24, 25]. We used the CHARMM program to set up the system and run the molecular dynamics for the first couple of hundred picoseconds and the rest of the simulation was run with the NAMD program. The DOPS lipid was built using the InsightII software (San Diego, California) and optimized in water. The lipid was then rotated randomly to generate a library of DOPS lipid for the construction of the membrane. The TIP3P water potential was used to model water molecules [26]. The system consists of 40 phospholipid molecules ( $\sim 70 \AA^{2} /$ lipid), one saposin $\mathrm{C}$ protein ( 8 net negative charges), 4597 water molecules, 56 sodium ions, and 8 chloride ions. The system was neutralized and contained about 100-150 mM salt. Depending on the number of protonated 
sites or neutralized amino termini, some sodium ions were taken out of or added into the simulation boxes to maintain neutral systems. The non-bonded van der Waals interactions and the electrostatic interactions were truncated and switched using a cutoff of $12 \AA$ in CHARMM. The particle mesh Ewald (PME) summation with no truncation for the electrostatic interactions was applied in NAMD with a grid of $64 \times 64 \times 200$. All H-bond were held fixed. Fluorescence quenching of Trp-saposin C using spin-labeled phosphatidylcholine indicated that saposin $\mathrm{C}$ was embedded in the membrane to a depth of $\sim 5$ carbon bond lengths [12]. Therefore, we used the monolayer membrane. The geometry of the simulation cell adopted the similar approach, as described elsewhere $[27,28]$. The cell in the $\mathrm{z}$ direction is arbitrarily chosen to be $200 \AA$. A vacuum layer was present on the top of the water layer. A wall potential was applied to prevent the diffusion of water molecules into vacuum. The system was built and simulated following the protocol proposed by Berneche et al. [29] All simulations were run in the NPT ensemble, in which the dimension of the central box was kept constant in the $\mathrm{x}-\mathrm{y}$ plane, while allowing fluctuations along the $\mathrm{z}$ axis.

No experimental data exists for the accurate position of saposin $\mathrm{C}$ bound in membrane. Studies have shown that $\mathrm{H}-1$ and $\mathrm{H}-5$ helices of saposin $\mathrm{C}$ were most likely embedded in the membrane $[12,30]$ and the Asn22 residue (saposin C's glycosylation site), located at the loop between $\mathrm{H}-1$ and $\mathrm{H}-2$, was not close to the protein-membrane binding area and solvent exposed [14]. Therefore, we decided to put the amino and carboxylate termini of saposin $\mathrm{C}$ slightly embedded and the tilt angle of the axis of H-1 and H-5 to the membrane at about $25^{\circ}$ initially. With the protein held fixed, 40 large $\mathrm{VdW}$ spheres were randomly positioned on the $\mathrm{x}-\mathrm{y}$ plane at $\mathrm{z}=0$. A molecular dynamics simulation of 5 picoseconds was carried out to allow the spheres to relax around the protein on the $x-y$ plane. The phospholipids were placed to form a monolayer with their polar head groups at the centers of the VdW spheres and their tails stretching in the negative $\mathrm{z}$ direction. The lipids were then translated and rotated to minimize bad contacts, defined as two heavy atoms being closer than $2.6 \AA$. Subsequently, a water layer of $50 \AA$ was put on the top of the membrane and water molecules were deleted if they overlapped with the existing atoms, defined as being closer than $2.6 \AA$. There were about 4600 water molecules in the simulations. The total number of atoms was about 20400. A harmonic potential $U_{\text {wall }}=10(\mathrm{z}-50)^{2} \mathrm{kcal} / \mathrm{mol}$ at $\mathrm{z}=50 \AA$ was used to prevent the bulk water molecules from diffusing in the positive direction. Finally, sodium and chloride ions were added to balance the net charge of the protein and maintain a salt concentration of $\sim 100-150 \mathrm{mM}$.

\section{RESULTS}

\section{pKa Calculation}

Fig. (2) displays the calculated apparent pKa values $v s$ dielectric constants for $6 \mathrm{Glu}$ and 2 Asp residues in the sequence of saposin $\mathrm{C}$ at different $\mathrm{pH}$ values. Also included in the graphs are results from a previous study [14], which measured $\mathrm{pKa}$ values for 7 out the $11 \mathrm{Glu}$ residues in saposin $\mathrm{C}$ in the range of $\sim 5.1-5.6$ (the solid straight line in Fig. (2)). The figures demonstrate that the best $\mathrm{pKa}$ values could be reproduced with the protein dielectric constants of 10-20. With the protein electric constant of 80 , the results were slightly worse. Demchuk and Wade studied the effect of the dielectric constant on $\mathrm{pKa}$ calculations and proposed two classes of titratable sites; mostly solvent exposed residues with a protein dielectric constant close to that of the aqueous solvent and mostly buried residues with a lower protein dielectric constant in the range of 10-20 [31]. Since all charged residues in saposin $\mathrm{C}$ are solvent exposed, the results from these calculations differ from the conclusion of Demchuk and Wade, but may still be within the uncertainty of the method.

Previous studies of saposin $\mathrm{C}$ and its binding with negatively charged membranes showed that the protein-vesicle binding occurred at acidic $\mathrm{pH}[9,14,32]$ and had an apparent binding $\mathrm{pKa}$ value of $\sim 5.3$ [14]. Further, the protein-vesicle binding was a reversible process controlled by $\mathrm{pH}$. The change of $\mathrm{pH}$ directly affects the protonation states of the titratable residues and hence the interactions between the membrane and the residues' side chains. As a result, the hydrophilicity of the protein surface changes.

\section{Molecular Dynamics Simulation}

To better understand the protein-vesicle binding, especially the role of $\mathrm{pH}$ in the reversible binding process, we have carried out two simulations, i.e. saposin $\mathrm{C}$ with residues in their default charge states and saposin $C$ with several neutralized Glu and Asp residues within the negatively charged membrane of DOPS. Simulations were run for about $8.5 \mathrm{~ns}$. A snapshot at the beginning of the simulations is shown in Fig. (3). The three disulfide bridges were kept intact during all simulations. The neutralized residues were chosen based on our calculated $\mathrm{pKa}$ values $>5.0$ in the low range of the dielectric constants of saposin C, namely, Glu6, Glu9, Glu25, Glu46, Glu69, Asp20, and Asp52.

The Positions of $\mathrm{C} \alpha$ atom of $\mathrm{Val} 3$ in the $\mathrm{z}$ direction relative to the average phosphorus atom's position at neutral and lower $\mathrm{pH}$ versus time are present in Fig. (4) for the convergence test. All average quantities were calculated from the last 2 ns trajectories. Saposin C has an electrostatic surface with a predominantly negative potential due to the high content of negatively charged residues and a few positively charged Lys residues located in the amino-terminal half of saposin $\mathrm{C}$, i.e. the $\mathrm{H}-1$ and $\mathrm{H}-2$ regions (Fig. 1b). Initially, the saposin $\mathrm{C}$ fold was oriented to have a tilt angle of $\sim 25^{\circ}$ (H1) to the membrane surface. The region having positive charges was closer to the membrane surface and the amino and carboxylate terminal parts were slightly embedded in the area of the lipid heads. Fig. (4) shows that the saposin C fold was relaxed from the initial position and equilibrated after about $4 \mathrm{~ns}$. After relaxation and equilibration (total length $=$ $8.5 \mathrm{~ns}$ ), the final tilt angles of $\mathrm{H}-1$ and $\mathrm{H}-5$ were $\sim 62^{\circ}$ and $\sim 45^{\circ}$ for the default charge case, and $\sim 40^{\circ}$ and $\sim 30^{\circ}$ for the case of neutralized residues. The final orientation is the balance of the electrostatic interactions between the charged residues in the protein and the polar groups of the lipids.

Since the relative orientation of saposin $\mathrm{C}$ depends on the interactions between the charged side chains of the protein residues and the charged groups of the lipids, the local environment around the charged groups was investigated. The 

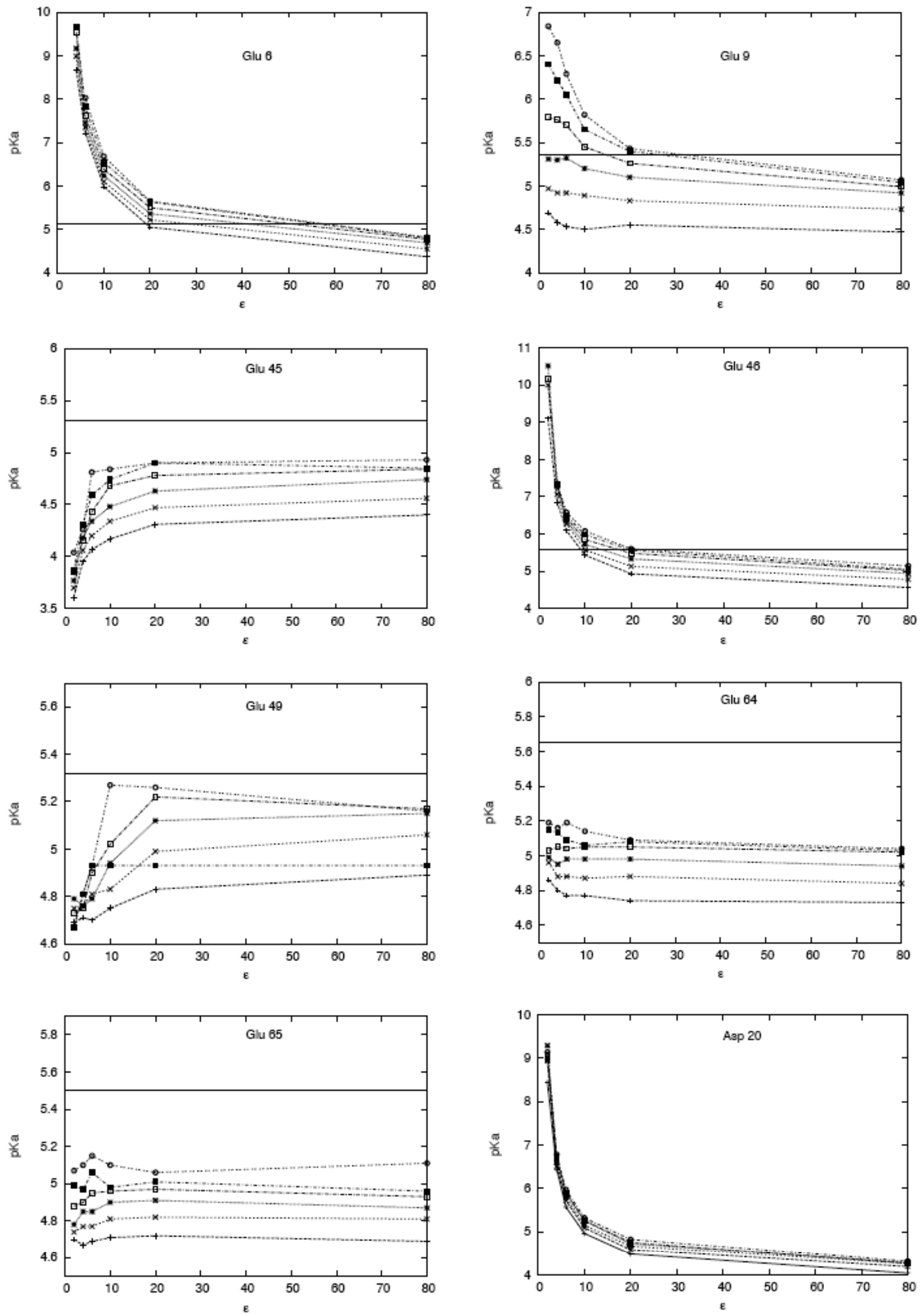

Fig. (2). $\mathrm{pKa} v s$ dielectric constant at different $\mathrm{pH}$ values for selected residues. — ; experimental data, $+; \mathrm{pH}=4.5, \times ; \mathrm{pH}=5.0, * ; \mathrm{pH}=$ $5.5, \square ; \mathrm{pH}=6.0, \mathbf{\square} ; \mathrm{pH}=6.5, \circ ; \mathrm{pH}=7.0$. 


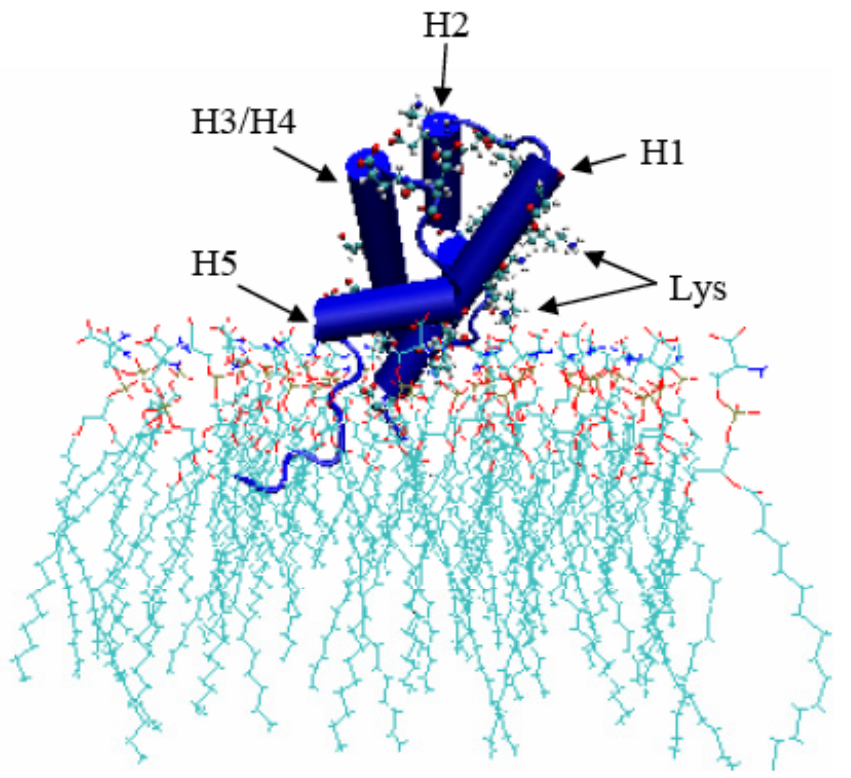

Fig. (3). Snapshot of the system at the initial position. Charged residues are presented in CPK form. Graphics were prepared using the VMD (Visual Molecular Dynamics) program.

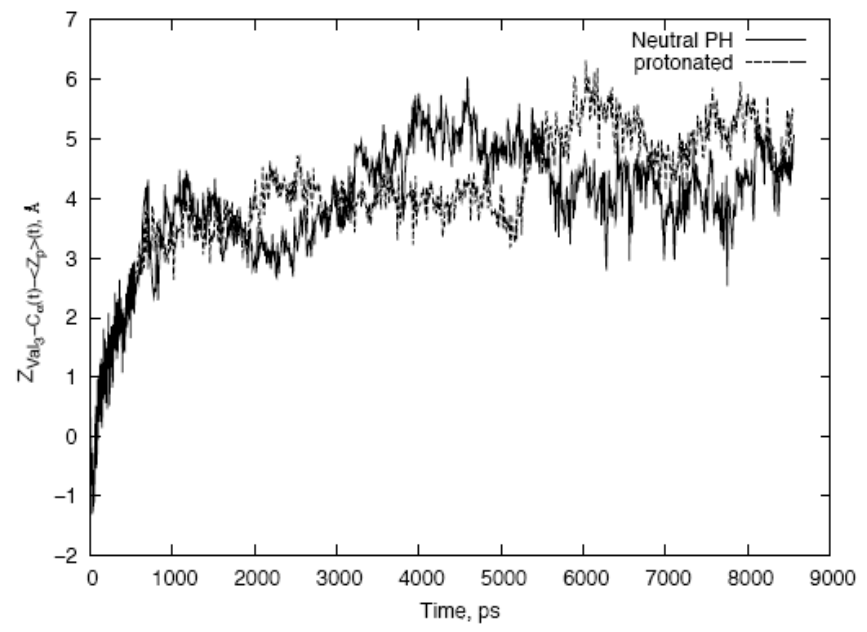

Fig. (4). Position of $\mathrm{C} \alpha$ atom of $\mathrm{Val} 3$ in the $\mathrm{z}$ direction relative to the average phosphorus atom's position $\left\langle z_{\mathrm{p}}>\right.$ with time, - default charge case, --- protonated case.

default charge environment corresponds to the system at neutral $\mathrm{pH}$, at which no protein-vesicle binding occurs. The positively charged side chains of the protein are thus farther away from the negatively charged membrane surface and the electrostatic interactions are weak. The radial distribution functions between the charged groups of the protein and the membrane reveal the $\mathrm{pH} /$ neutralization effect in more detail. In Fig. (5), the radial distribution functions of several selected residues and the charge groups of the membrane are presented. Fig. (5a-c) shows the radial distribution functions between the negatively charged Glu residues with the positively charged amino termini of the lipids in both cases. The figure shows that the distances between the Glu residues and amino groups are much smaller in the default charge environment (solid curves) than at low $\mathrm{pH}$ (dashed curves). The first peaks of the solid curves (radial distributions between the oxygen atoms of the glutamate side chains and the nitrogen atoms of the amino groups of the lipids) occur at $\sim 2.8 \AA$ for Glu6, $\sim 7 \AA$ for Glu9, and $\sim 3.5 \AA$ for Glu45, respectively, whereas at low $\mathrm{pH}$ the first peaks of the dashed curves are $\sim 6.2 \AA$ for Glu6, $\sim 12 \AA$ for Glu9, and $\sim 14.2 \AA$ for Glu45, respectively. This means that the attractive interactions between the negatively charged Glu residues and the positively charged amino groups of the lipids are very strong at a neutral $\mathrm{pH}$. These interactions prevent the protein from penetrating into the membrane. The strength of the attractive interactions decreases with a decrease in $\mathrm{pH}$, due to the partial neutralization of the Glu residues.

In contrast to the negatively charged residues, the positively charged side chains of Lys residues moved closer to the negatively charged carboxylate groups of the lipid heads at low $\mathrm{pH}$ (Fig. 5d,e), the dashed curves shifted to the left], which means that the attractive interactions between the positively charged Lys residues of the protein and the negatively charged carboxylate groups of the lipids increases with the decrease in $\mathrm{pH}$ because of the reduction of the attractive interactions in the opposite direction. These interactions promote the protein's penetration into the membrane. In addition, the reduction of the negative-negative repulsion helps strengthen the attractive interaction between the Lys residues and the membrane. The tilt angles of the $\mathrm{H}-1$ and $\mathrm{H}-5$ helices reflected the balance of the different interactions. If the interactions between the Lys residues and the membrane increase, the tilt angles of the H-1 and H-5 helices will decrease. The interaction energies of the Lys 13 side chain with the membrane were $-0.01 \pm 0.01 \mathrm{kcal} / \mathrm{mol}$ and $-0.17 \pm 0.2$ $\mathrm{kcal} / \mathrm{mol}$ for the systems with default charges and some protonated residues, respectively. It is interesting to notice the Lys41 residue in Fig. (5f) is located in the loop between the $\mathrm{H}-2$ and H-3 helices. Lys41 strongly binds to the oxygen atoms of the lipid phosphate group and is embedded in the membrane. This changed slightly with the charge states of other residues and played a role in the anchoring of the carboxylate terminal of $\mathrm{H}-2$ and the amino terminal of $\mathrm{H}-3$ of the protein on the membrane. It seems that Lys41 and Lys38, besides Lys13, also play an important role in protein/membrane binding and protein conformational change. Protein-vesicle binding depends on environmental variables such as $\mathrm{pH}$, composition of the membrane, and ionic strength, etc. Also, it is a slow process in the computer simulation timescale. Some phenomena may not be observed during a simulation on the order of nanoseconds. Nevertheless, the simulation results beautifully depict the $\mathrm{pH}$ controlled reversibility of the binding process of saposin $\mathrm{C}$ on a negatively charged membrane.

The sodium counterions were mainly distributed in the range of the lipid heads and shielded the negatively charged groups of the membrane. The radial distribution functions of sodium ions around the negatively charged groups are presented in Fig. (5g). The chloride ions mainly shielded the positively charged side chains of the Lys residues (Fig. 5h). Snapshots of the systems at the end of the simulations are present in Fig. (6). 

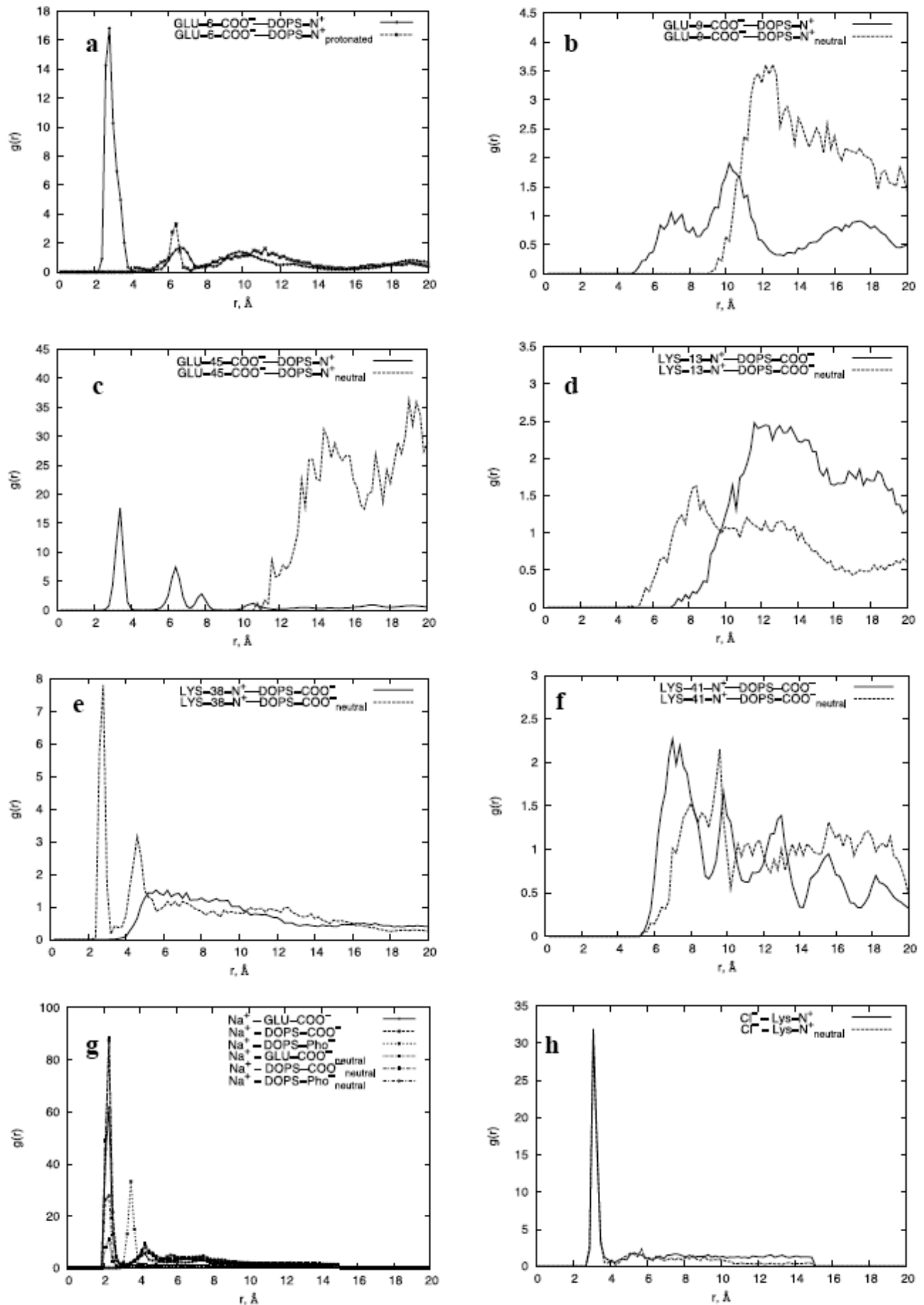

Fig. (5). Radial distribution functions of charged atoms of saposin C with respect to charged atoms of DOPS; (a) Glu6, (b) Glu9, (c) Glu45, (d) Lys13, (e) Lys38, (f) Lys41, and ions with charged atoms in saposin C as well as DOPS; (g) Sodium ions with charged oxygen atoms, (h) chloride ions with charged nitrogen atoms of Lys residues. - with default charge states at neutral $\mathrm{pH}$, --- with some neutralized residues (lower $\mathrm{pH}$ ). 
(a)

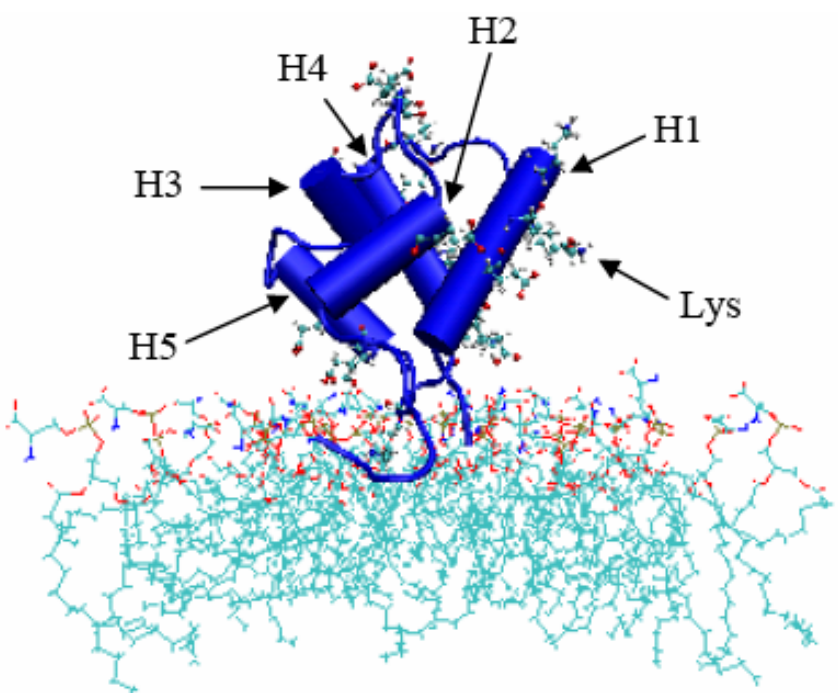

(b)

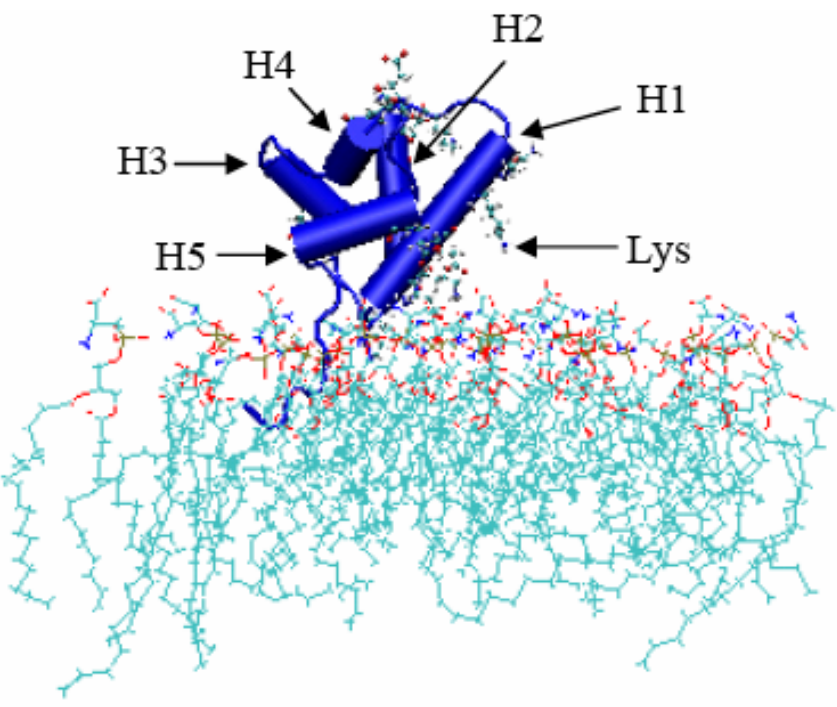

Fig. (6). Snapshots of the systems at the end of the simulations; (a) system at neutral $\mathrm{pH}$ and (b) system with some neutralized residues.

\section{Simulations in Modified DOPS}

Since the positively charged groups of the lipids play a very important role in the low $\mathrm{pH}$ binding process, the saposin $\mathrm{C} / \mathrm{membrane}$ binding can be expected to be independent of the $\mathrm{pH}$ if there are no positively charged groups in the lipids, as in the detergent SDS [13]. Two simulations were carried out in the default charge state to examine the role of the positively charged groups in the saposin Cmembrane binding. In one simulation, the amino and carboxylate groups of the lipids were all neutralized, but the total negatively charged groups (phosphorus groups) were the same as in the unmodified case. All other conditions were kept the same as in the previous simulations. In the second simulation, only the amino groups of the lipids were neutralized, in which the negatively charged groups $\left(\mathrm{COO}^{-}\right.$ and $\mathrm{PO}_{4}{ }^{-}$) doubled. Forty sodium ions were added to keep the system neutral. Both simulations were about $1.2 \mathrm{~ns}$ long. The relative short simulations were intended to show qualitatively or semi-quantitatively the affects by modifying the charged terminal groups. The positions of $\mathrm{C} \alpha$ atom of $\mathrm{Val} 3$ in the $\mathrm{z}$ direction relative to the average phosphorus atom's position $\left\langle\mathrm{z}_{\mathrm{p}}\right\rangle$ with time in the modified DOPS are presented in Fig. (7). The relative small fluctuations of the positions of Val3 show that saposin $\mathrm{C}$ binds strongly to the membrane, especially in the modified lipid- $\mathrm{NH}_{3}{ }^{+}$-termini only case. The radial distribution functions between the oxygen atoms of the Lys side chains and the nitrogen atoms of the amino groups of the lipids are presented in Fig. $(\mathbf{8 a}, \mathbf{b})$. The radial distribution functions were calculated from the last 500 ps trajectories. Snapshots at the end of the simulation for saposin C in DOPS with modified terminal groups are presented in Fig. (9) for graphic comparison of the conformational change. From the radial distribution functions in Fig. (8) we can see that the side chains of Lys13, Lys38, and Lys41 are very close to the negatively charged groups of the lipids and most likely form hydrogen bonds. The interaction energies of the Lys13 side chain with the membrane were $1.40 \pm 0.86 \mathrm{kcal} / \mathrm{mol}$ and $-32.72 \pm 1.99 \mathrm{kcal} / \mathrm{mol}$ for the lipids with both $\mathrm{N}$-terminus and C-terminus neutralized and only $\mathrm{N}$-terminus neutralized, respectively. The $\mathrm{N}$-terminus of $\mathrm{H}-1$ inserted into the membrane in both cases. The results indicate that the electrostatic repulsion between the negative surfaces of the protein and the membrane is less important in the binding process, whereas the attractive interactions are dominant. The major effect of the partial neutralization of the negatively charged electrostatic surface of the protein by reducing $\mathrm{pH}$ is to decrease the attractive interaction between the negative electrostatic surface of the protein and the positive charge groups of the lipids. This increases the attractive interaction between the positively charged Lys residues and the negatively charged carboxylate groups of the lipids because it enables the Lys side chains to more closely approach the membrane surface. The two pairs of attractive interactions act mainly on the opposite sides of the surface, depicted as the front and back views of the electrostatic surface of the protein in Fig. (1b). The orientation of the protein, represented through the tilt angle of the $\mathrm{H}-1$ helix, is mainly determined by these two pairs of interactions.

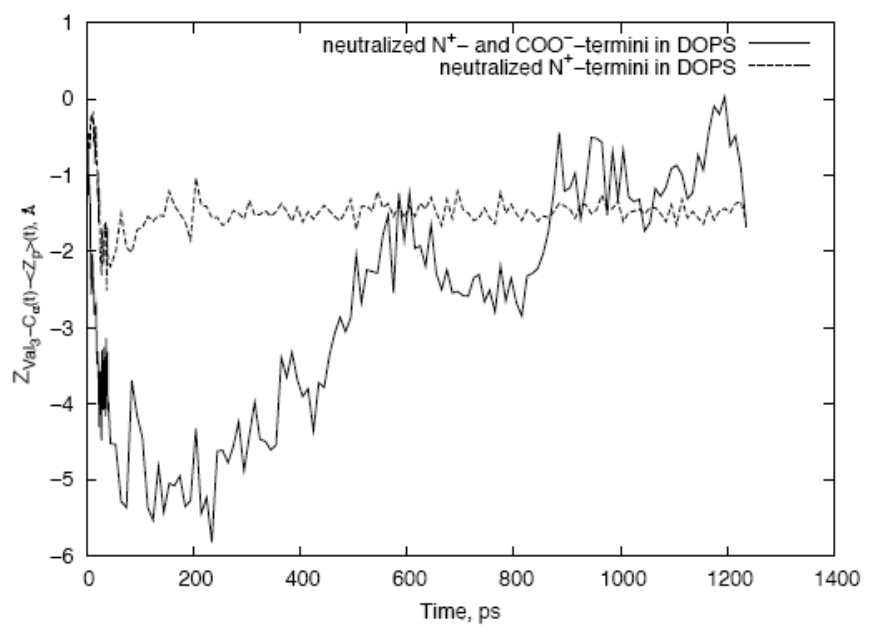

Fig. (7). Position of $\mathrm{C} \alpha$ atom of $\mathrm{Val} 3$ in the $\mathrm{z}$ direction relative to the average phosphorus atom's position $\left\langle\mathrm{z}_{\mathrm{p}}\right\rangle$ with time in modified DOPS. 

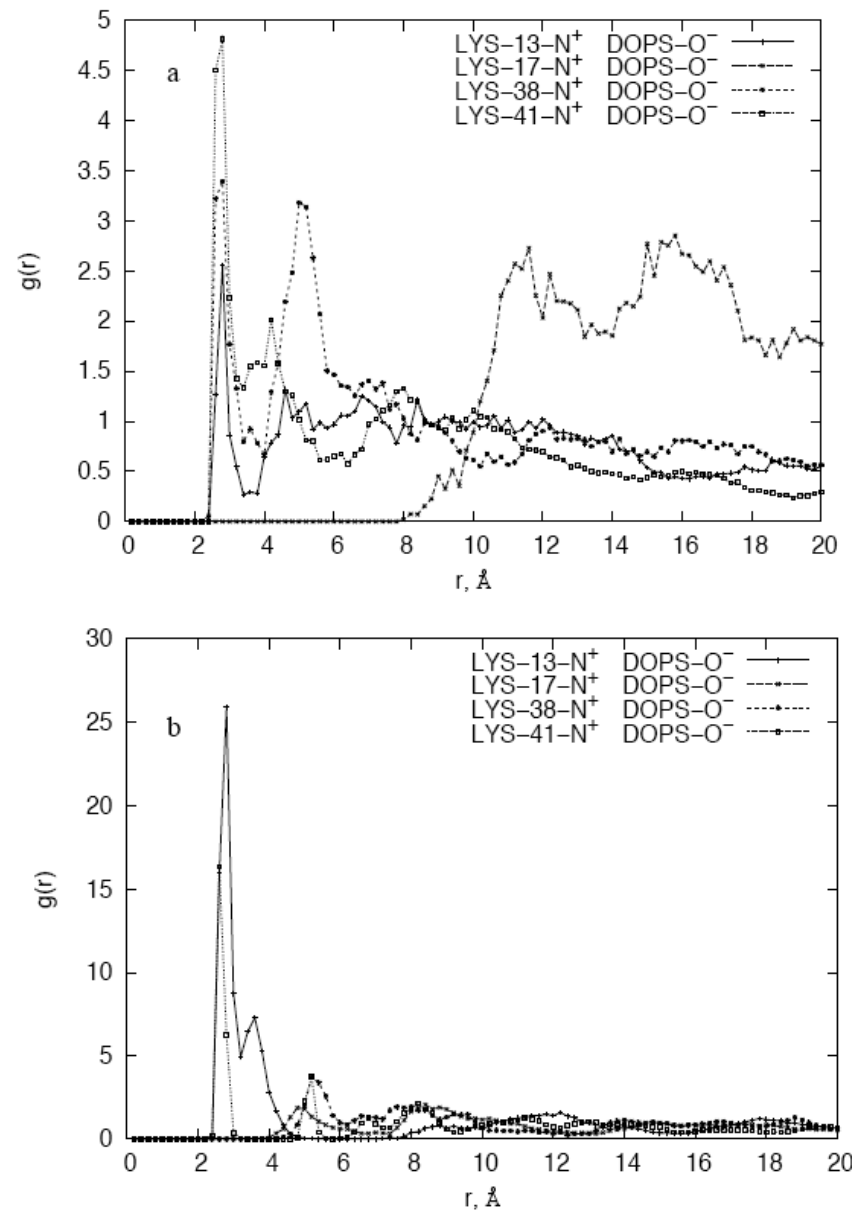

Fig. (8). The radial distribution functions of the nitrogen atoms in the Lys side chains and the oxygen atoms in negatively charged groups of modified lipids; (a) N-terminus and C-terminus of DOPS were neutralized, (b) only the N-terminus of DOPS was neutralized.

Our previous study showed that the Lys residues played an important role in the membrane anchoring of saposin $\mathrm{C}$ [5]. Therefore, the surface on which the Lys residues locate has to bend toward the membrane surface to allow binding to occur. When the attractive interactions between the positive amino groups of the lipids and the negative electrostatic surface of the protein are strong enough to pull the protein up and separate the Lys side chains from the membrane, saposin $\mathrm{C}$ release from the membrane probably occurs. Without the positively charged groups in the membrane, membrane binding should be independent of $\mathrm{pH}$ or less dependent on $\mathrm{pH}$. According to past studies $[9,12]$, membrane binding leads to a conformational change in saposin $\mathrm{C}$, and the middle region of saposin $\mathrm{C}$ is exposed to the aqueous phase and not embedded in the membrane, which was also confirmed by the study from Hawkins et al. [13]. On the other hand, de Alba et al. didn't observe the conformational change in their NMR study of the behavior of saposin $\mathrm{C}$ as a function of $\mathrm{pH}$ [14], but found the conformation changed to a U-shape in detergent [13]. Comparison of Fig. (9) with Figs. (3) and (6) shows that a part of the fourth helix unfolded and that the amino terminal of the third helix rose up from the membrane surface, which may indicate the opening of a hydrophobic pocket. The insertion of the $\mathrm{H}-1$ helix into the membrane and (a)

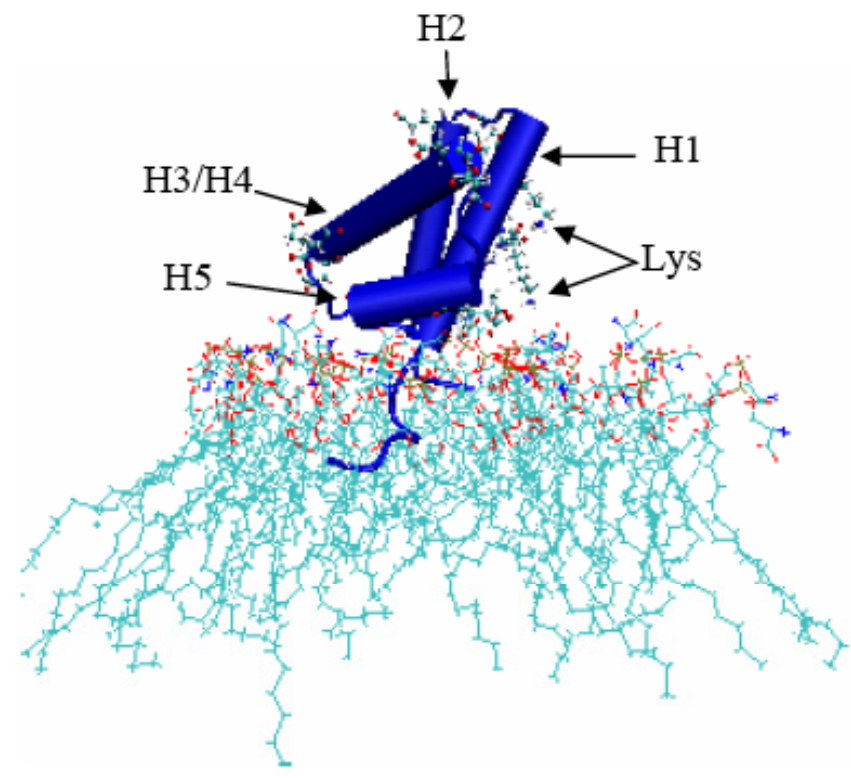

(b)

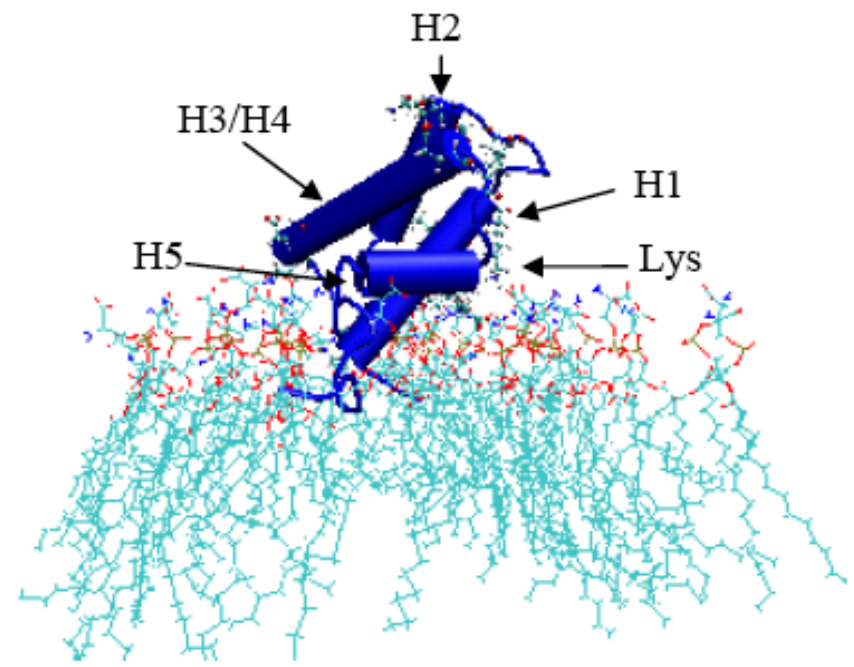

Fig. (9). Snapshots of saposin C in modified DOPS; (a) both amino and carboxylate groups neutralized and (b) only amino group neutralized. Charged residues are shown in CPK form. Graphics were prepared using the VMD program.

the anchoring of the loop between the H-2 and $\mathrm{H}-3$ helices on the membrane by Lys38 and Lys41 likely resulted in the opening of the hydrophobic core. The calculated order parameters $-\mathrm{S}_{\mathrm{CD}}$ of the hydrocarbon chains are presented in Fig. (10). The hydrocarbon chains have average values of $0.2-$ 0.07 for $\mathrm{C} 2-\mathrm{C} 8$ and $0.0-0.05$ for $\mathrm{C} 11-\mathrm{C} 17$, respectively, except in the DOPS- $\mathrm{NH}_{3}{ }^{+}$neutralized system, where the chains have average values of $\sim 0.15$ for $\mathrm{C} 11-\mathrm{C} 17$. The more structured or tilted tails most likely occurred due to the deeper insertion of saposin $\mathrm{C}$ and the strong interactions between saposin $\mathrm{C}$ and the lipids. This demonstrates that saposin $\mathrm{C}$ is a membrane-perturbing and lipid-binding protein, which results in membrane curvature changes. Membrane curvature is regarded as a prime player in growth, division, and movement [33]. 


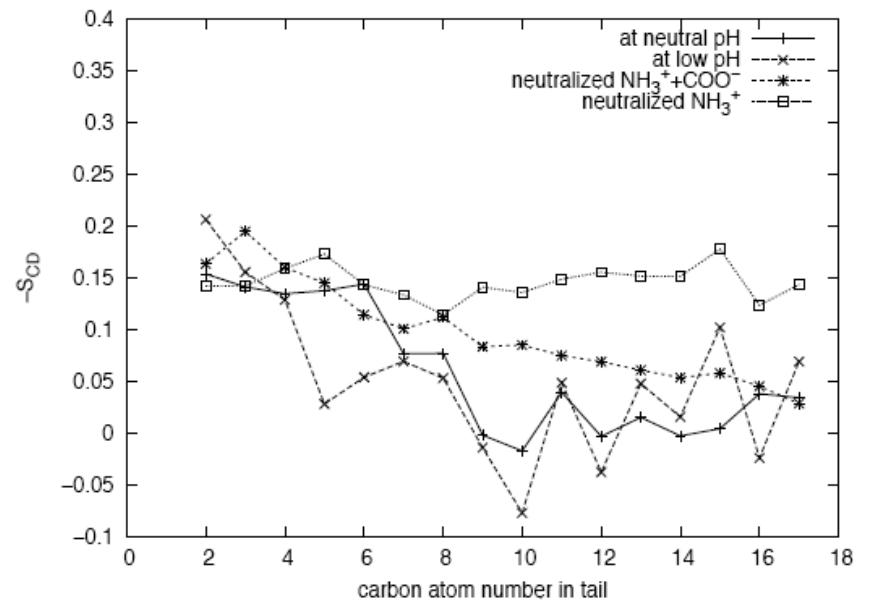

Fig. (10). Order parameters $S_{C D}$ of the hydrocarbon chains for the unmodified and modified DOPS.

\section{DISCUSSION}

We have performed several simulations of saposin $\mathrm{C}$ with the default charge states at neutral $\mathrm{pH}$ and some neutralized amino acid residues at acidic $\mathrm{pH}$ in normal and modified DOPS, respectively. The residues chosen to be neutralized in the simulation were based on the $\mathrm{pKa}$ calculations using the UHBD program. The neutralized residues applied in the simulations did not include all residues with elevated pKa's measured in the experiments, and the $\mathrm{pKa}$ calculations did not consider the effect of the membrane. In saposin $C$, the Glu residues with elevated $\mathrm{pKa}$ are located in three clusters on the protein surface; Glu6 - Glu9, Glu45 - Glu46 Glu49, and Glu64 - Glu65. The calculated pKa values using the single site model are lower than the experimental values for Glu45, Glu49, Glu64, and Glu65 either with lower or higher dielectric constants. This may indicate the limitation of the single site model for strongly coupled titratable sites, which may need the multiple site titration treatment $[34,35]$.

Saposin C-membrane binding is mainly the result of the balance of the electrostatic interactions between the charged groups in the protein and those in the membrane lipids. Certainly, the hydrophobic effect and the shielding effect of counter ions and salt may also influence protein-membrane binding, but may not be the major factors under the conditions of this study. The change of $\mathrm{pH}$ mainly affects the protonation states of negatively charged residues, which in turn shift the electrostatic balanced position of the protein on the membrane. From the calculated interaction energies of the Lys 13 side Lys side chains and the membrane. With a decrease of $\mathrm{pH}$, the interaction increased. Without the Glumembrane attractive interaction, the Lys residues interacted strongly with the membrane. The simulation results elucidated the mechanism of the $\mathrm{pH}$ controlled reversible binding process of saposin $\mathrm{C}$ on a negatively charged membrane. In a study using atomic force microscopy of phospholipid membrane interactions with saposin $\mathrm{C}[11,30]$, two types of membrane restructuring over time were observed; formation of patch-like structural domains and the occurrence of membrane destabilization. The protein surface neutralization near the membrane surface is a crucial step for the above processes to occur with membranes containing positively charged amino groups. We believe that the two pairs of attractive electrostatic interactions play a dominant role in the $\mathrm{pH}$ controlled membrane binding process.

The details of saposin C-DOPS interactions, upon which the results and conclusions rest, depend perhaps critically on the orientation and position of the protein within the membrane, although our previous simulation study showed that the initial conformations of saposin C molecule didn't affect the final binding [5]. One more challenge is to study systems containing anionic phospholipids such as PG, which has only negatively charged groups, as does SDS. Saposin C-SDS binding is independent of $\mathrm{pH}$, whereas saposin $\mathrm{C}-\mathrm{PG}$ binding depends on $\mathrm{pH}$ [36-38]. SDS has a more flexible membrane curvature that may also affect the interplay between SDS and saposin C. A possible explanation for the $\mathrm{pH}$ dependent saposin C-PG binding is that the balance of the electrostatic interactions are between the attractive interactions of Saposin $\mathrm{C}-\mathrm{N}^{+}-\mathrm{PG}^{-} \mathrm{O}^{-}$and the repulsive interactions of saposin $\mathrm{C}^{-} \mathrm{O}^{-}$ - $\mathrm{PG}_{-}^{-}$, which mainly act on the same surface (the front side in Fig. (1b)). Probably, the reduction of the negativenegative repulsion by protonation of negatively charged residues is still important in those systems so that the positively charged residues can closely approach the membrane surface. Since cellular membranes contain less than $30 \%$ of anionic lipids, we expect a significant decrease in saposin Cmembrane repulsion. It will promote saposin $\mathrm{C}$ and lipid membrane binding. More detailed study is needed to reveal the nature of the saposin C-membrane binding in those systems.

\section{CONLUSIONS}

The major effect of the charge neutralization of saposin $\mathrm{C}$ by reduction of the $\mathrm{pH}$ is to reduce the electrostatic attraction between the negatively charged residues of the protein and the positively charged amino groups of the lipids. Certainly, the charge neutralization on the protein surface reduces the electrostatic repulsion between the protein surface and the membrane, which makes the Lys side chains more easily able to approach the membrane. Therefore, the simulation results reveal that the repulsive interactions between the protein and membrane play a less important role in membrane binding. The present study provides insight into the nature of the $\mathrm{pH}$ controlled reversible binding process of saposin $\mathrm{C}$ to membrane, i.e. the $\mathrm{pH}$ value controls the interactions between the negatively charged Glu residues of the protein with the positively charged amino termini of the lipids. The final binding is realized through the interactions between the positively charged Lys residues with the negatively charged groups in the membrane. Since the electrostatic and hydrophobic interactions of saposin $\mathrm{C}$ depend on the protein environment and the membrane composition, future studies will use membranes with anionic lipids and different lipid compositions. Studies of residue mutations will be pivotal to identify important individual interactions in structure and functionality of saposin $\mathrm{C}$ in enzyme stimulation.

\section{REFERENCES}

[1] Hiraiwa M, Martin BM, Kishimoto Y, Conner GE, Tsuji S, O'Brien JS. Lysosomal proteolysis of prosaposin, the precursor of saposins (sphingolipid activator proteins); its mechanism and inhibition by ganglioside. Arch Biochem Biophys 1997; 341; 17-24.

[2] Hiraiwa M, Soeda S, Martin BM, et al. The effect of carbohydrate removal on stability and activity of saposin B. Arch Biochem Biophys $1993 ; 303 ; 326-31$. 
[3] Kishimoto Y, Hiraiwa M, O'Brien JS. Saposins; structure, function, distribution, and molecular genetics. J Lipid Res 1992; 33; 125567.

[4] Liepinsh E, Andersson M, Ruysschaert JM, Otting G. Saposin fold revealed by the NMR structure of NK-lysin. Nat Struct Biol 1997; 4; 793-5.

[5] Liu A, Wenzel N, Qi X. Role of lysine residues in membrane anchoring of saposin C. Arch Biochem Biophys 2005; 443; 101-12.

[6] Qi X, Leonova T, Grabowski GA. Functional human saposins expressed in Escherichia coli. Evidence for binding and activation properties of saposins $\mathrm{C}$ with acid beta-glucosidase. J Biol Chem 1994; 269; 16746-53

[7] Regis S, Filocamo M, Corsolini F, et al. An Asn > Lys substitution in saposin B involving a conserved amino acidic residue and leading to the loss of the single N-glycosylation site in a patient with metachromatic leukodystrophy and normal arylsulphatase A activity. Eur J Hum Genet 1999; 7; 125-30.

[8] Sandhoff K, Kolter T, Harzer K. Sphingolipid Activator Proteins. In; Scriver CR, Beaudet AL, Sly WS, Valle D, eds. The Metabolic and Molecular Bases of Inherited Disease. Eighth ed. New York; McGraw-Hill, Inc. 2000; 3371-88.

[9] Vaccaro AM, Salvioli R, Barca A, et al. Structural analysis of saposin $\mathrm{C}$ and B. Complete localization of disulfide bridges. J Biol Chem 1995; 270; 9953-60.

[10] Wang Y, Grabowski GA, Qi X. Phospholipid vesicle fusion induced by saposin C. Arch Biochem Biophys 2003; 415; 43-53.

[11] You HX, Yu L, Qi X. Phospholipid membrane restructuring induced by saposin $\mathrm{C}$; a topographic study using atomic force microscopy. FEBS Lett 2001; 503; 97-102.

[12] Qi X, Grabowski GA. Differential membrane interactions of saposins $\mathrm{A}$ and $\mathrm{C}$; implications for the functional specificity. $\mathrm{J}$ Biol Chem 2001; 276; 27010-7.

[13] Hawkins CA, Alba E, Tjandra N. Solution structure of human saposin $\mathrm{C}$ in a detergent environment. J Mol Biol 2005; 346; 138192 .

[14] de Alba E, Weiler S, Tjandra N. Solution structure of human saposin $\mathrm{C}$; $\mathrm{pH}$-dependent interaction with phospholipid vesicles. Biochemistry 2003; 42; 14729-40.

[15] Madura JD, Briggs JM, Wade RC, et al. Electrostatics and diffusion of molecules in solutions; simulations with the University of Houston Brownian Dynamics Program. J Comput Phys Commun 1995; 91; 57-95

[16] Antosiewicz J, McCammon JA, Gilson MK. The determinants of pKas in proteins. Biochemistry 1996; 35; 7819-33.

[17] Gibas CJ, Subramaniam S. Explicit solvent models in protein $\mathrm{pKa}$ calculations. Biophys J 1996; 71; 138-47.

[18] Oberoi H, Allewell NM. Multigrid solution of the nonlinear Poisson-Boltzmann equation and calculation of titration curves. Biophys J 1993; 65; 48-55.

[19] Pethig R. Protein-water interactions determined by dielectric methods. Ann Rev Phys Chem 1992; 43; 177-205.

[20] Schutz CN, Warshel A. What are the dielectric "constants" of proteins and how to validate electrostatic models? Proteins 2001; 44; 400-17.
[21] Simonson T, Perahia D, Bricogne G. Intramolecular dielectric screening in proteins. J Mol Biol 1991; 218; 859-86.

[22] Sternberg MJ, Hayes FR, Russell AJ, Thomas PG, Fersht AR. Prediction of electrostatic effects of engineering of protein charges. Nature $1987 ; 330 ; 86-8$.

[23] Warshel A, Russell ST. Calculations of electrostatic interactions in biological systems and in solutions. Quart Rev Biophys 1984; 17; 283-422.

[24] Brooks B. Meeting the educational needs of health care personnel.. twenty-four hours a day. Am J Infect Control 1983; 11; 30A-1A.

[25] Lu H, Schulten K. Steered molecular dynamics simulations of force-induced protein domain unfolding. Proteins 1999; 35; 45363.

[26] Jorgensen WL, Chandrasekhar J, Medura JD, Impey RW, Klein ML. Comparison of simple potential functions for simulation liquid water. J Chem Phys 1983; 79; 926-935.

[27] Kaznessis YN, Kim S, Larson RG. Simulations of zwitterionic and anionic phospholipid monolayers. Biophys J 2002; 82; 1731-42.

[28] Kaznessis YN, Kim S, Larson RG. Specific mode of interaction between components of model pulmonary surfactants using computer simulations. J Mol Biol 2002; 322; 569-82.

[29] Berneche S, Nina M, Roux B. Molecular dynamics simulation of melittin in a dimyristoylphosphatidylcholine bilayer membrane. Biophys J 1998; 75; 1603-18.

[30] You HX, Qi X, Grabowski GA, Yu L. Phospholipid membrane interactions of saposin $\mathrm{C}$; in situ atomic force microscopic study. Biophys J 2003; 84; 2043-57.

[31] Demchuk E, Wade RC. Improving the continuum dielectric approach to calculation $\mathrm{pKa}$ of ionizable groups in proteins. J Phys Chem 1996; 100; 17373-17387.

[32] Vaccaro AM, Tatti M, Ciaffoni F, Salvioli R, Serafino A, Barca A. Saposin $\mathrm{C}$ induces $\mathrm{pH}$-dependent destabilization and fusion of phosphatidylserine-containing vesicles. FEBS Lett 1994; 349; 181 6.

[33] McMahon HT, Gallop JL. Membrane curvature and mechanisms of dynamic cell membrane remodelling. Nature 2005; 438; 590-6.

[34] Beroza P, Fredkin DR, Okamura MY, Feher G. Protonation of interacting residues in a protein by a Monte Carlo method; application to lysozyme and the photosynthetic reaction center of Rhodobacter sphaeroides. Proc Natl Acad Sci USA 1991; 88; $5804-$ 8.

[35] Gilson MK. Multiple-site titration and molecular modeling; two rapid methods for computing energies and forces for ionizable groups in proteins. Proteins $1993 ; 15 ; 266-82$.

[36] Abu-Baker S, Qi X, Lorigan GA. Investigating the interaction of saposin C with POPS and POPC phospholipids; a solid-state NMR spectroscopic study. Biophys J 2007; 93; 3480-90.

[37] Abu-Baker S, Qi X, Newstadt J, Lorigan GA. Structural changes in a binary mixed phospholipid bilayer of DOPG and DOPS upon saposin $\mathrm{C}$ interaction at acidic $\mathrm{pH}$ utilizing (31)P and (2)H solidstate NMR spectroscopy. Biochim Biophys Acta 2005; 1717; 5866.

[38] Qi X, Grabowski GA. Acid beta-glucosidase; intrinsic fluorescence and conformational changes induced by phospholipids and saposin C. Biochemistry $1998 ; 37 ; 11544-54$.

(C) Liu and Qi; Licensee Bentham Open.

This is an open access article distributed under the terms of the Creative Commons Attribution License (http://creativecommons.org/licenses/by/2.5/), which permits unrestrictive use, distribution, and reproduction in any medium, provided the original work is properly cited. 